\title{
Dynamic renormalization group analysis of propagation of elastic waves in two-dimensional heterogeneous media
}

\author{
Reza Sepehrinia ${ }^{1}$, Alireza Bahraminasab ${ }^{1,2}$, Muhammad Sahimi $^{3}{ }^{*}$ and M. Reza Rahimi Tabar ${ }^{1,4,5}$ \\ ${ }^{1}$ Department of Physics, Sharif University of Technology, Tehran 11365-9161, Iran \\ ${ }^{2}$ Department of Physics, Lancaster University, Lancaster LA1 4 YB, United Kingdom \\ ${ }^{3}$ Mork Family Department of Chemical Engineering Materials Science, University of Southern California, \\ Los Angeles, California 90089-1211, USA \\ ${ }^{4}$ Observatoire de la Côte d'Azur, CNRS UMR 6529, Boîte Postale 4229, 06304 Nice Cedex 4, France \\ ${ }^{5}$ Carl Institute of Physics, von Ossietzky University, D-26111 Oldenburg, Germany
}

\begin{abstract}
We study localization of elastic waves in two-dimensional heterogeneous solids with randomly distributed Lamé coefficients, as well as those with long-range correlations with a power-law correlation function. The Matin-Siggia-Rose method is used, and the one-loop renormalization group (RG) equations for the the coupling constants are derived in the limit of long wavelengths. The various phases of the coupling constants space, which depend on the value $\rho$, the exponent that characterizes the power-law correlation function, are determined and described. Qualitatively different behaviors emerge for $\rho<1$ and $\rho>1$. The Gaussian fixed point (FP) is stable (unstable) for $\rho<1(\rho>1)$. For $\rho<1$ there is a region of the coupling constants space in which the RG flows are toward the Gaussian FP, implying that the disorder is irrelevant and the waves are delocalized. In the rest of the disorder space the elastic waves are localized. We compare the results with those obtained previously for acoustic wave propagation in the same type of heterogeneous media, and describe the similarities and differences between the two phenomena.
\end{abstract}

PACS numbers: 62.30.+d, 47.56.+r, 05.10.Cc, 71.23.An

\section{INTRODUCTION}

Ever since the discovery of electron localization, ${ }^{1}$ much attention has been devoted to this phenomenon, since it is not only of fundamental scientific interest, but also has much practical importance. There is now extensive experimental evidence for the localization phenomenon in disordered materials. ${ }^{2,3}$ On the theoretical side, the problem has been studied for decades by several analytical methods, ranging from the scaling theory ${ }^{4}$ to the selfconsistent perturbation theory. ${ }^{5,6}$ In addition, numerical simulations using such techniques as the transfer-matrix method and the statistics of energy levels have been used to verify the predictions of the analytical results.

The development of the one-parameter scaling theor ${ }^{4}$ of electron localization in terms of the concepts of critical phenomena suggests that, the problem can be reformulated by using an effective field theory which, when done, leads to the so-called $\sigma$ model which is a nonlinear model. Wegner ${ }^{7}$ proposed such a description of disordered conductors. Also noteworthy among the theoretical developments is the work of Efetov et al.,$^{8}$ who proposed the supersymmetric approach, now used widely. The renormalization group (RG) approach, one of the most powerful methods in statistical physics, has also been used to examine the critical properties of the resulting effective field theory. ${ }^{9}$ The RG approach leads to a set of equations for the coupling constants, such as the diffusivity and conductance of the disordered materials under study. The main prediction of all of these approaches is that, for space dimensions $d>2$, there is a transition from the localized to extended states, so that the lower critical dimension of the localization phenomenon is, $d_{c}=2$.
However, despite convincing numerical evidence for the validity of this prediction, ${ }^{10}$ the exponent $\nu$ that characterizes the power-law behavior of the localization length $\xi$ near the phase transition, $\xi \propto\left|W-W_{c}\right|^{-\nu}$ (where $W_{c}$ is the critical value of the disorder intensity $W$ ), predicted by the RG method, is not in agreement with the numerical results. A possible explanation for this discrepancy is that, some of the terms that are neglected in the construction of the field theory may actually be relevant to the RG analysis.

Another approach to the field-theoretic description of the problem is based on the method first developed by Martin, Siggia, and Rose (MSR), ${ }^{11}$ by which one constructs an effective action (see below) based on the governing stochastic equation of motion for the phenomenon under study. The MSR approach is well developed for critical phenomena far from equilibrium, ${ }^{11}$ and has been extensively used to study various dynamical critical phenomena, such as those that are described by the Langevin equation, or the driven interface phenomena, such as surface growth and stochastic hydrodynamics. The main advantages of the MSR method are that, it provides an exact generating functional, and that one needs no approximation in order to obtain the effective action. This is the method that we use in the present paper.

An important implication of the wave characteristics of electrons is that, the localization phenomenon may also occur in propagation of the classical waves in disordered media. However, unlike the problem of electron localization in strongly disordered materials, classical waves, such as seismic waves, ${ }^{12,13}$ do not interact with each other and, therefore, their propagation in heterogeneous media 
provides an ideal model for studying the phenomenon of localization of the classical waves. Moreover, along with the work on electronic transport in disordered materials, parallel work has been carried out on localization properties of classical waves in disordered media that describe the phonons that are responsible for heat transport in solids. ${ }^{14}$

Although waves that are described by scalar equations have been used for describing phonons in disordered materials, a more suitable continuum description of the phenomenon is through propagation of elastic waves. Due to the presence of different polarizations and the coupling between them (mode conversion), propagation of elastic waves in disordered solids constitutes a complex set of phenomena. ${ }^{15,16}$ Because of this complexity, there have been relatively few studies in the literature dealing with propagation of elastic waves in disordered solid. ${ }^{17}$ In particular, localization of special types of elastic waves has been studied in the past, ranging from surface elastic (Rayleigh) waves, ${ }^{18}$ to transverse deflections of a beam, ${ }^{19}$ and coherent backscattering and multiple scattering. ${ }^{20,21}$

At the same time, understanding how elastic waves propagate at very large scales, particularly in highly heterogeneous media such as rock, is fundamental to a host of other important problems, such as earthquakes, underground nuclear explosions, the morphology and content of oil and gas reservoirs, oceanography, and materials sciences. ${ }^{12}$ For example, seismic wave propagation and reflection are used to not only estimate the hydrocarbon content of a potential oil or gas field and gain insight into its morphology, but also to image structures located over a wide area, ranging from the Earth's near surface to the deeper crust and upper mantle. ${ }^{22}$

The purpose of the present paper is to study the effect of heterogeneities, represented by spatial distributions of the local elastic constants, on elastic wave propagation in disordered media, such as rock, which represents a highly heterogeneous natural material. Recently, extensive experimental data for the spatial distributions of the local elastic moduli, the density, and the wave velocities in several large-scale porous rock formations, both off- and onshore, were analyzed. ${ }^{23}$ The analysis provided strong evidence for the existence of long-range correlations in the spatial distributions of the measured quantities, characterized by a power-law correlation function. The existence of such correlations in the data provided the impetus for the present study and motivated an important question that we address in the present paper: how do large-scale heterogeneities and long-range correlations affect elastic wave propagation in disordered media? Another question that we address in the present paper is whether, in the presence of the heterogeneities, the elastic waves can be delocalized. By localization we mean a situation in which, over finite length scales (which can, however, be large), the waves' amplitude decays and essentially vanishes.

Localization of elastic waves in rock would imply, for example, that seismic exploration yields useful informa- tion only over distances $r$ from the explosion's site that are of the order of the localization length $\xi$. Thus, if, for example, $\xi$ is on the order of a few kilometers, but the linear size of the area for which a seismic exploration is done is significantly larger than $\xi$, then, seismic recordings can, at best, provide only partial information about the area. Localization of elastic waves also implies that, if the stations that collect data for seismic waves that are emanated from an earthquake in rock are farther from the earthquake's hypocenter than $\xi$, no useful information on the seismic activity prior to and during the earthquake can be gleaned from the data ${ }^{24}$.

We use a field-theoretic formulation to study propagation of elastic waves in two-dimensional (2D) disordered media in which the Lamé coefficients are spatially distributed. Our approach is based on the MSR method. ${ }^{11}$ We calculate the one-loop $\beta$ functions (see below) for both spatially random and power-law correlated distribution ${ }^{23}$ of the local elastic constants. Although our work is primarily motivated by the analysis of experimental data for the spatial distribution of elastic constants of rock at large scales ${ }^{23}$ the results presented in this paper are general and applicable to any solid material in which the local elastic constants follow the statistics of the distributions that we consider. The present paper represents the continuation of our previous work $\mathrm{w}^{25,26}$ in which we studied acoustic wave propagation in the same type of heterogeneous media. We will compare the results with those obtained previously for propagation of acoustic waves.

The rest of this paper is organized as follows. In Sec. II the model is described and the governing equations are presented. Section III describes the field-theoretic description of the elastic wave equation, and the development of the MSR formulation for the propagation of the waves in heterogeneous media. In Sec. IV the perturbative RG calculations, based on the MSR action, are carried out and the results are analyzed. In Sec. V we compare the results with those obtained previously ${ }^{25,26}$ for propagation of acoustic waves in the same type of heterogeneous media that we consider in the present paper. The paper is summarized in Sec. VI.

\section{THE MODEL AND GOVERNING EQUATIONS}

To analyze propagation of elastic waves in a disordered medium, we begin with the equation of motion of an elastic medium with the mean density $m$,

$$
m \frac{\partial^{2} u_{i}}{\partial t^{2}}=\partial_{j} \sigma_{i j}
$$

where $u_{i}$ is the displacement in the $i$ th direction, and $\sigma_{i j}$ the $i j$ th component of the stress tensor $\boldsymbol{\sigma}$. As usual, $\sigma_{i j}$ is expressed in terms of the strain tensor,

$$
\sigma_{i j}(\mathbf{x})=2 \mu(\mathbf{x}) u_{i j}+\lambda(\mathbf{x}) u_{k k} \delta_{i j}
$$


For small deformations, the strain tensor is given by,

$$
u_{i j}=\frac{1}{2}\left(\partial_{i} u_{j}+\partial_{j} u_{i}\right)
$$

where $\lambda$ and $\mu$ are the Lamé coefficients. For simplicity, we take the two Lamé coefficients to be equal, but the main results of the paper presented below will not change if they are unequal, but follow the same type of statistical distributions. Hence, we write,

$$
\mu(\mathbf{x})=\lambda(\mathbf{x})=\lambda_{0}+\eta(\mathbf{x}),
$$

where $\lambda_{0}=\langle\lambda(\mathbf{x})\rangle$, with $\langle\cdot\rangle$ representing a spatial averaging. We assume that $\eta(\mathbf{x})$, the fluctuating part of the
Lamé coefficients, is a Gaussian random process. Thus, in performing the spatial average over the disorder we use a probability distribution of the form

$$
P[\eta(\mathbf{x})] \propto \exp \left[-\int d \mathbf{x} d \mathbf{x}^{\prime} \eta(\mathbf{x}) D\left(\mathbf{x}-\mathbf{x}^{\prime}\right) \eta\left(\mathbf{x}^{\prime}\right)\right]
$$

where $D(\mathbf{x})$ is the inverse of the correlation function $C(\mathbf{x})$. The disorder that we include in the model consists of two parts. One is (random) $\delta$-correlated, while the second part is characterized by a power-law correlation function. Hence, the overall correlation function of the spatial distribution of the disorder is given by

$$
\left\langle\eta(\mathbf{x}) \eta\left(\mathbf{x}^{\prime}\right)\right\rangle=2 C\left(\mathbf{x}-\mathbf{x}^{\prime}\right)=2 D_{0} \delta^{d}\left(\mathbf{x}-\mathbf{x}^{\prime}\right)+2 D_{\rho}\left|\mathbf{x}-\mathbf{x}^{\prime}\right|^{2 \rho-d}
$$

in which $D_{0}$ and $D_{\rho}$ are, respectively, the strengths of the disorder for the random and the power-law correlated parts, $C\left(\mathbf{x}-\mathbf{x}^{\prime}\right)$ satisfies the following condition

$$
\int d \mathbf{x}^{\prime \prime} C\left(\mathbf{x}-\mathbf{x}^{\prime \prime}\right) D\left(\mathbf{x}^{\prime \prime}-\mathbf{x}^{\prime}\right)=\delta\left(\mathbf{x}-\mathbf{x}^{\prime}\right)
$$

and $d$ is the spatial dimension ( $d=2$ in this paper). Note that, in 2D, $\rho=H+1$, with $H$ being the Hurst exponent.

A Gaussian distribution of the form (5) gives rise to quadratic couplings in the interaction part of the action defined below. Moreover, the Gaussian distribution (5) may include a tail of inadmissible negative values of the Lamé coefficients. In principle, the unphysical tail can be removed by introducing a modified probability distribution function which, however, would produce couplings of higher order in the action. But, interactions of orders higher than quadratic are irrelevant in the $\mathrm{RG}$ analysis and, therefore, can be ignored.

We now take the Fourier transform of Eq. (1) with respect to the time variable, which yields the governing equation for a monochromatic wave with angular frequency $\omega$,

$$
\partial_{j} \sigma_{i j}+\omega^{2} m u_{i} \equiv \lambda_{0} \mathcal{L}_{i j} u_{j}=0
$$

Here, $\mathcal{L}$ is a $2 \times 2$ differential matrix operator (see below).

\section{FIELD-THEORETIC REPRESENTATION OF THE ELASTIC WAVE EQUATION}

Using the formalism developed by De Dominicis and Peliti ${ }^{27}$ (see also Hochberg et $a .^{28}$ ), one obtains a MSR generating functional that corresponds to the (Fouriertransformed) wave equation (8)

$$
\begin{aligned}
P\left[u_{i}^{R}, u_{i}^{I}\right] & =\frac{1}{\mathcal{N}} \int[\mathcal{D} \eta]\left[\mathcal{D}\left\{u_{i}^{R}, u_{i}^{I}\right\}\right] \delta\left(\mathcal{L}_{1 j} u_{j}^{R}\right) \delta\left(\mathcal{L}_{2 j} u_{j}^{R}\right) \delta\left(\mathcal{L}_{1 j} u_{j}^{I}\right) \delta\left(\mathcal{L}_{2 j} u_{j}^{I}\right) \\
& \times J\left(\frac{\partial \mathcal{L} \mathbf{u}^{R}}{\partial \mathbf{u}^{R}}\right) J\left(\frac{\partial \mathcal{L} \mathbf{u}^{I}}{\partial \mathbf{u}^{I}}\right) \exp \left[-\int d \mathbf{x} d \mathbf{x}^{\prime} \eta(\mathbf{x}) D\left(\mathbf{x}-\mathbf{x}^{\prime}\right) \eta\left(\mathbf{x}^{\prime}\right)\right] .
\end{aligned}
$$

Here, superscripts $R$ and $I$ indicate, respectively, the real and imaginary parts of the solution of the wave equation, $J$ is the Jacobian and, $\mathcal{N}$ is a normalization constant.
The Jacobian for the transformation, $\mathbf{u} \rightarrow \mathcal{L} \mathbf{u}$, is expressed as a Grassman integral over the anticommuting fields $\chi_{i}, \chi_{i}^{*}$,

$$
J=\int \mathcal{D}\left\{\chi_{i}^{*}, \chi_{i}\right\} \exp \left\{\int d x\left[\chi_{1}^{*}(\mathbf{x}) \chi_{2}^{*}(\mathbf{x})\right]\left(\begin{array}{cc}
\mathcal{L}_{11} & \mathcal{L}_{12} \\
\mathcal{L}_{21} & \mathcal{L}_{22}
\end{array}\right)\left[\begin{array}{l}
\chi_{1}(\mathbf{x}) \\
\chi_{2}(\mathbf{x})
\end{array}\right]\right\}
$$


We now introduce two other auxiliary fields, $\tilde{u}_{i}^{R}$ and $\tilde{u}_{i}^{I}$, in order to express the $\delta$-functions in Eq. (9) as Fourier transforms. Then, substituting Eq. (10) in Eq. (9) and integrating out $\eta$, (by performing a Gaussian integration) leads to an effective MSR action $S_{e}$ with the following form

$$
\begin{aligned}
S_{e} & =S_{0}+S_{I} \\
S_{0} & =\int d \mathbf{x} \sum_{a=R, I}\left[i \tilde{\mathbf{u}}^{a}(\mathbf{x}) \cdot \mathcal{L}_{0} \mathbf{u}^{a}(\mathbf{x})+\chi^{a *}(\mathbf{x}) \cdot \mathcal{L}_{0} \chi^{a}(\mathbf{x})\right] \\
S_{I} & =\int d \mathbf{x} d \mathbf{x}^{\prime}\left[\sum_{a=R, I} i \partial_{j} \tilde{u}_{i}^{a}\left(2 \partial_{i} u_{j}^{a}+2 \partial_{j} u_{i}^{a}+\partial_{k} u_{k}^{a} \delta_{i j}\right)+\partial_{j} \chi_{i}^{a *}\left(2 \partial_{i} \chi_{j}^{a}+2 \partial_{j} \chi_{i}^{a}+\partial_{k} \chi_{k}^{a} \delta_{i j}\right)\right]_{\mathbf{x}} \\
& \times \frac{C\left(\mathbf{x}-\mathbf{x}^{\prime}\right)}{2 \lambda_{0}^{2}}\left[\sum_{a=R, I} i \partial_{j} \tilde{u}_{i}^{a}\left(2 \partial_{i} u_{j}^{a}+2 \partial_{j} u_{i}^{a}+\partial_{k} u_{k}^{a} \delta_{i j}\right)+\partial_{j} \chi_{i}^{a *}\left(2 \partial_{i} \chi_{j}^{a}+2 \partial_{j} \chi_{i}^{a}+\partial_{k} \chi_{k}^{a} \delta_{i j}\right)\right]_{\mathbf{x}^{\prime}},
\end{aligned}
$$

where the subscripts $\mathbf{x}$ and $\mathbf{x}^{\prime}$ indicate where the quantities are evaluated at. The explicit form of the matrix $\mathcal{L}_{0}$ is given by

$$
\mathcal{L}_{0}=\left(\begin{array}{cc}
3 \partial_{x}^{2}+\partial_{y}^{2}+\omega^{2} / \lambda_{0} & 2 \partial_{x} \partial_{y} \\
2 \partial_{x} \partial_{y} & \partial_{x}^{2}+3 \partial_{y}^{2}+\omega^{2} / \lambda_{0}
\end{array}\right) .
$$

We now write down the action in the Fourier space and, then, introduce a change of the basis to decouple the free propagator into two components, the longitudinal and transverse propagators. To do so, we use a transformation $\mathbf{A} \rightarrow \mathbf{U A}$ in order to diagonalize the matrix $\mathcal{L}_{0}$ in the Fourier space, where it has the following form,

$$
\mathcal{L}_{0}=\left(\begin{array}{cc}
-3 k_{x}^{2}-k_{y}^{2}+\omega^{2} / \lambda_{0} & -2 k_{x} k_{y} \\
-2 k_{x} k_{y} & -k_{x}^{2}-3 k_{y}^{2}+\omega^{2} / \lambda_{0}
\end{array}\right)
$$

with following eigenvectors

$$
|1\rangle=\frac{1}{k}\left(\begin{array}{c}
k_{x} \\
k_{y}
\end{array}\right), \quad|2\rangle=\frac{1}{k}\left(\begin{array}{c}
-k_{y} \\
k_{x}
\end{array}\right) .
$$

The corresponding eigenvalues are $\left(\omega^{2} / \lambda_{0}-k^{2}\right)$ and $\left(\omega^{2} / \lambda_{0}-3 k^{2}\right)$, respectively. The two eigenvalues represent the dispersion relations of the transverse and longitudinal waves which propagate in a uniform medium with the phase velocities, $v_{t}=\sqrt{\lambda_{0}}$ and $v_{l}=\sqrt{3 \lambda_{0}}$. Using the eigenvectors, the transformation matrix $\mathbf{U}$ is given by

$$
\mathbf{U}=\frac{1}{k}\left(\begin{array}{cc}
k_{x} & -k_{y} \\
k_{y} & k_{x}
\end{array}\right)
$$

By applying the transformation $\mathbf{U}$, we finally obtain

$$
\begin{aligned}
S_{0}=\int_{k} \sum_{a=R, I}\left[i \tilde{\mathbf{u}}^{a}(-k) \cdot \mathcal{L}_{0}^{d} \mathbf{u}^{a}(k)+\chi^{a *}(-k) \cdot \mathcal{L}_{0}^{d} \chi^{a}(k)\right] \\
S_{I}=\int_{k, p_{i}}\left[\sum_{a=R, I} i \tilde{\mathbf{u}}^{a}\left(p_{1}\right) \cdot \mathcal{L}_{I} \mathbf{u}^{a}\left(p_{2}\right)+\chi^{a *}\left(p_{1}\right) \cdot \mathcal{L}_{I} \chi^{a}\left(p_{2}\right)\right]\left[g_{0} \delta\left(\sum_{i} p_{i}\right)+g_{\rho} k^{-2 \rho} \delta\left(p_{1}+p_{2}-k\right) \delta\left(p_{3}+p_{4}+k\right)\right] \\
\times\left[\sum_{a=R, I} i \tilde{\mathbf{u}}^{a}\left(p_{3}\right) \cdot \mathcal{L}_{I} \mathbf{u}^{a}\left(p_{4}\right)+\chi^{a *}\left(p_{3}\right) \cdot \mathcal{L}_{I} \chi^{a}\left(p_{4}\right)\right]
\end{aligned}
$$

with

$$
\mathcal{L}_{0}^{d}=\left(\begin{array}{cc}
-k^{2}+\omega^{2} / \lambda_{0} & 0 \\
0 & -3 k^{2}+\omega^{2} / \lambda_{0}
\end{array}\right), \quad \mathcal{L}_{I}=\left(\begin{array}{cc}
A\left(p_{1}, p_{2}\right) & -C\left(p_{1}, p_{2}\right) \\
C\left(p_{1}, p_{2}\right) & B\left(p_{1}, p_{2}\right)
\end{array}\right)
$$

where,

$$
\begin{aligned}
& A=c\left[3\left(p_{1} \cdot p_{2}\right)^{2}+\left|p_{1} \times p_{2}\right|^{2}\right], \\
& B=c\left[\left(p_{1} \cdot p_{2}\right)^{2}-\left|p_{1} \times p_{2}\right|^{2}\right], \\
& C=c\left[2\left(p_{1} \cdot p_{2}\right)\left(p_{1} \times p_{2}\right) \cdot \hat{z}\right],
\end{aligned}
$$


to the $(x y)$ plane. Here the $p_{i}(i=x, y)$ represent $2 \mathrm{D}$ wave vectors that span the square $\left\{\left|p_{x}\right|,\left|p_{y}\right|<\Lambda\right\}$ in the Fourier space, for which we have adopted the standard convention by defining

$$
\int_{p}=\int_{-\Lambda}^{\Lambda} \int_{-\Lambda}^{\Lambda} d^{2} p
$$

Two coupling constants, $g_{0}=D_{0} / \lambda_{0}^{2}$ and $g_{\rho}=D_{\rho} / \lambda_{0}^{2}$, appear in $S_{e}$, for which we carry out an RG analysis in the limit, $\omega^{2} / \lambda_{0} \rightarrow 0$, in order to derive, to one loop, the $\beta$ functions that describe their behavior in the coupling space. Note that for those terms of $S_{I}$ with symmetric products of the fields under an exchange of momenta, the corresponding coefficients will also retain the symmetric part. For example, the coefficient of $g_{0} \tilde{u}_{1}^{R}\left(p_{1}\right) u_{1}^{R}\left(p_{2}\right) \tilde{u}_{1}^{R}\left(p_{3}\right) u_{1}^{R}\left(p_{4}\right)$ is written as a sum of the symmetric and antisymmetric parts,

$$
\begin{aligned}
A\left(p_{1}, p_{2}\right) A\left(p_{3}, p_{4}\right)=\frac{1}{2}[ & \left.A\left(p_{1}, p_{2}\right) A\left(p_{3}, p_{4}\right)+A\left(p_{1}, p_{2}\right) A\left(p_{3}, p_{2}\right)\right] \\
& +\frac{1}{2}\left[A\left(p_{1}, p_{2}\right) A\left(p_{3}, p_{4}\right)-A\left(p_{1}, p_{2}\right) A\left(p_{3}, p_{2}\right)\right]
\end{aligned}
$$

so that the antisymmetric part is cancelled by integrating over the momenta.

\section{RENORMALIZATION GROUP ANALYSIS}

To study whether the elastic waves are localized or delocalized in the $2 \mathrm{D}$ heterogeneous media of the type that we consider, we apply the RG method to the effective action, Eq. (16). To do so, we follow the momentum shell $\mathrm{RG}^{29,30}$ and sum over the short wavelength degrees of freedom. More specifically, we denote all the fields in the action (16) by $\Phi(k)$. To facilitate the analysis, we change the domain of the integration from the square to a circle of radius $\Lambda$. Since the small $k$ modes are supposed to control the critical behavior of the system in the vicinity of localization-delocalization transition, the change does not make any qualitative difference to the results. Here- after, we refer to the small $k$ modes as the slow modes, and the rest as the fast modes. We then define two sets of variables

$$
\begin{aligned}
& \Phi_{<}=\Phi(k) \text { for } 0<k<\Lambda / l, \text { slow modes } \\
& \Phi_{>}=\Phi(k) \text { for } \Lambda / l \leq k \leq \Lambda, \text { fast modes }
\end{aligned}
$$

where $l>1$ is the rescaling parameter of the RG transformation. Then, the action is expressed in terms of $\Phi_{<}$ and $\Phi_{>}$as

$$
S\left(\Phi_{<}, \Phi_{>}\right)=S_{0}\left(\Phi_{<}\right)+S_{0}\left(\Phi_{>}\right)+S_{I}\left(\Phi_{<}, \Phi_{>}\right) .
$$

$S_{0}$ is a quadratic function of its arguments that can be separated into slow and fast terms, but $S_{I}$ mixes the two modes. Then, the partition function $Z$ is separated and written as follows

$$
Z=\int\left[\mathcal{D} \Phi_{<}\right] \int\left[\mathcal{D} \Phi_{>}\right] \exp \left[S_{0}\left(\Phi_{<}\right)\right] \exp \left[S_{0}\left(\Phi_{>}\right)\right] \exp \left[S_{I}\left(\Phi_{<}, \Phi_{>}\right)\right] \equiv \int\left[\mathcal{D} \Phi_{<}\right] \exp \left[S_{0}^{\prime}\left(\Phi_{<}\right)\right]
$$

which defines the effective action $S^{\prime}\left(\Phi_{<}\right)$for the slow modes:

$$
\begin{aligned}
\exp \left[S^{\prime}\left(\Phi_{<}\right)\right] & =\exp \left[S_{0}\left(\Phi_{<}\right)\right] \int\left[\mathcal{D} \Phi_{>}\right] \exp \left[S_{0}\left(\Phi_{>}\right)\right] \exp \left[S_{I}\left(\Phi_{<}, \Phi_{>}\right)\right] \\
& =\exp \left[S_{0}\left(\Phi_{<}\right)\right] \int\left[\mathcal{D} \Phi_{>}\right] \exp \left[S_{0}\left(\Phi_{>}\right)\right] \frac{\int\left[\mathcal{D} \Phi_{>}\right] \exp \left[S_{0}\left(\Phi_{>}\right)\right] \exp \left[S_{I}\left(\Phi_{<}, \Phi_{>}\right)\right]}{\int\left[\mathcal{D} \Phi_{>}\right] \exp \left[S_{0}\left(\Phi_{>}\right)\right]} \\
& \left.=Z_{0>} \exp \left[S_{0}\left(\Phi_{<}\right)\right] \exp \left[S_{I}\left(\Phi_{<}, \Phi_{>}\right)\right]\right\rangle_{0>},
\end{aligned}
$$



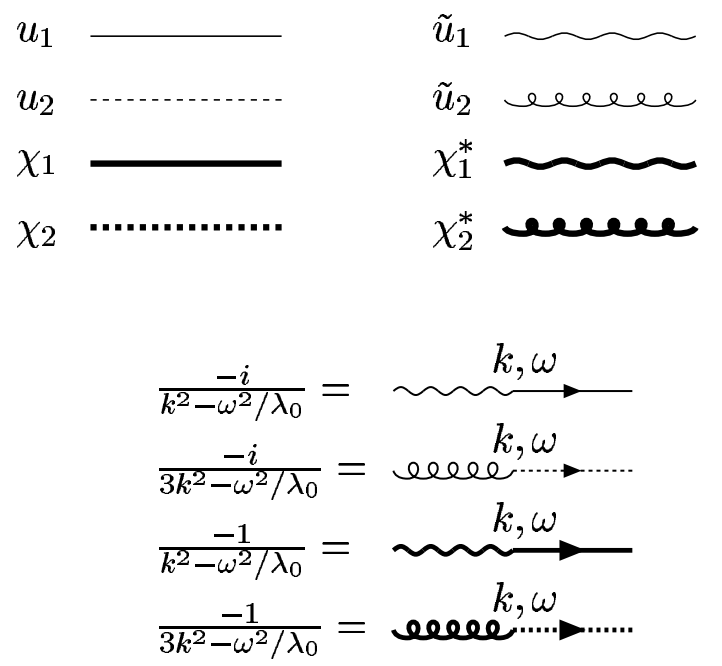

FIG. 1: Graphical representation of the fields and the Feynman rules for the propagators.

adds a constant to the action, independent of $\Phi_{<}$. The next step is to calculate the average $\left\langle\exp \left[S_{I}\left(\Phi_{<}, \Phi_{>}\right)\right]\right\rangle_{0>}$, which we treat perturbatively for weak disorder using the relation

$$
\langle\exp (V)\rangle=\exp \left[\langle V\rangle+\frac{1}{2 !}\left(\left\langle V^{2}\right\rangle-\langle V\rangle^{2}\right)+\cdots\right] .
$$

Therefore, according to Eqs. (18) and (19), we have, up to one-loop order

$$
S^{\prime}\left(\Phi_{<}\right)=\left\langle S_{I}\right\rangle+\frac{1}{2 !}\left(\left\langle S_{I}^{2}\right\rangle-\left\langle S_{I}\right\rangle^{2}\right) .
$$

Each term in the series contains some monomials in the fast and slow modes. The former must be averaged with respect to $S_{0}\left(\Phi_{>}\right)$. The first term in Eq. (20) yields tree-level terms, as well as the corrections to the kinetic term of $S_{0}$. We introduce a graphical representation of the terms which is shown in the Fig. 1. The Feynman diagrams that contribute to the kinetic term of the propagator $\tilde{u}_{1}^{R} u_{1}^{R}$ are shown in the Fig. 2. According to Fig. 2 , apart from a naive dimensional rescaling, one should rescale the fields by a factor $F$ in the following way, in order to keep the coefficient of the kinetic term to be the same as in the original action

$$
\Phi \rightarrow \frac{1}{\sqrt{F}} \Phi
$$

with

$$
F=1-18 \pi\left(\Lambda^{2} g_{0}+\Lambda^{-2 \rho+2} g_{\rho}\right) \delta l+\mathcal{O}\left(g_{0}^{2}, g_{\rho}^{2}, g_{0} g_{\rho}\right),
$$

where $\delta l=l-1$.

We now derive the RG equations for the disorder strengths by renormalization of the coupling of the vertex $\tilde{u}_{1}^{R}\left(p_{1}\right) u_{1}^{R}\left(p_{2}\right) \tilde{u}_{1}^{R}\left(p_{3}\right) u_{1}^{R}\left(p_{4}\right)$. The Feynman diagrams

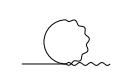

(r) $R, I: 4,2$

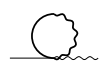

(r) $R, I: 2,2$

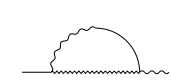

(r) $R: 2$

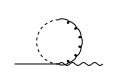

(r) $R, I: 2,2$

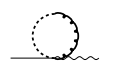

(r6) $R, I: 2,2$

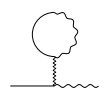

(r) $R, I: 2,2$

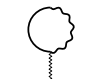

(r) $R, I: 2,2$

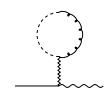

(r) $R, I: 2,2$

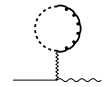

(r) $R, I: 2,2$
FIG. 2: Feynman diagrams for renormalization of the kinetic term $-i \tilde{u}_{1}^{R}(-k) k^{2} u_{1}^{R}(k)$ of $S_{0}$. They appear in the cumulant expansion to the lowest order. External legs are the slow modes, while the internal fields are the fast modes, and the integration is done over the fast modes. Those fields that cosist of loops can be real or imaginary, and are denoted by $R$ and $I$, respectively. The number of choices for the construction of each diagram is also shown. The diagrams with long-range interactions (zigzag lines) are divergent, due to the zero momentum carried by the zigzag lines, but such diagrams are canceled by the corresponding diagrams with Grassmanian loops (thick lines). In fact, only $r_{1}$ and $r_{9}$ contribute.

that contribute to the renormalized coupling in one-loop order of the perturbation expansion, and the corresponding symmetry factors, are shown in the Fig. 3. Note that the couplings are functions of the momenta and, therefore, we consider the first term in the Taylor expansion and set the external momenta to be parallel. The expressions for all the Feynman diagrams are listed in the Appendix. It can be seen by dimensional analysis that the canonical dimensions of the couplings in units of length are

$$
\begin{aligned}
& {\left[g_{0}\right]=2,} \\
& {\left[g_{\rho}\right]=2-2 \rho .}
\end{aligned}
$$

The following rules should be considered in expressing the Feynman diagrams of the vertex function shown in Fig. 3:

(i) The diagrams that are made by different vertices have an extra factor of 2 , due to the quadratic term of the cumulant expansion.

(ii) All the diagrams have a factor $1 / 2$ ! due to the cumulant expansion.

(iii) An extra (-1) factor should be included for the diagrams with Grassmanian loop.

Given the above rules, we obtain the following renormalized couplings

$$
\begin{array}{r}
g_{0}^{\prime} l^{2} \Lambda^{\prime 2}=F^{-2}\left(g_{0} \Lambda^{2}+\frac{937}{18} \pi g_{0}^{2} \Lambda^{4} \delta l+\frac{716}{27} \pi g_{\rho}^{2} \Lambda^{-4 \rho+4} \delta l\right. \\
\left.+\frac{3635}{54} \pi g_{0} g_{\rho} \Lambda^{-2 \rho+4} \delta l\right) \\
g_{\rho}^{\prime} l^{-2 \rho+2} \Lambda^{\prime-2 \rho+2}=F^{-2}\left(g_{\rho} \Lambda^{-2 \rho+2}+36 \pi g_{0} g_{\rho} \Lambda^{-2 \rho+4} \delta l\right. \\
\left.+\frac{1948}{27} g_{\rho}^{2} \Lambda^{-4 \rho+4} \delta l\right),(26)
\end{array}
$$




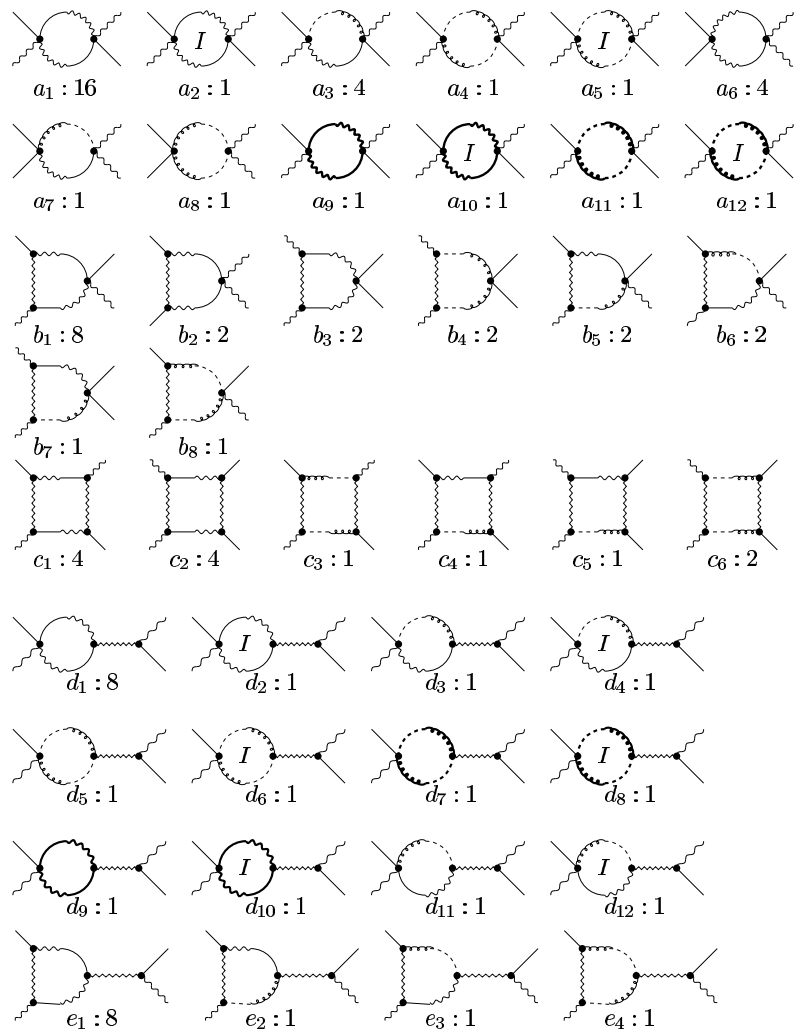

FIG. 3: Feynman diagrams for the renormalization of $\tilde{u}_{1}^{R}\left(p_{1}\right) u_{1}^{R}\left(p_{2}\right) \tilde{u}_{1}^{R}\left(p_{3}\right) u_{1}^{R}\left(p_{4}\right)$ in the action. The diagrams with loops of imaginary fields are indicated with $I$. The number of choices for constructing each diagram is also given.

where $\Lambda^{\prime}=\Lambda / l$. Using Eq. (22) and writing the equations in differential forms, we obtain the following expressions for the $\beta$ functions that describe the couplings

$$
\begin{aligned}
\beta\left(\tilde{g}_{0}\right) \equiv \frac{\partial \tilde{g}_{0}}{\partial \ln l}= & -2 \tilde{g}_{0}+\left(36+\frac{937}{18}\right) \tilde{g}_{0}^{2}+\frac{716}{27} \tilde{g}_{\rho}^{2} \\
& +\left(36+\frac{3635}{54}\right) \tilde{g}_{0} \tilde{g}_{\rho} \\
\beta\left(\tilde{g}_{\rho}\right) \equiv \frac{\partial \tilde{g}_{\rho}}{\partial \ln l}= & (2 \rho-2) \tilde{g}_{\rho}+72 \tilde{g}_{0} \tilde{g}_{\rho} \\
& +\left(36+\frac{1948}{27}\right) \tilde{g}_{\rho}^{2},
\end{aligned}
$$
by

where $\tilde{g}_{0}$ and $\tilde{g}_{\rho}$ are dimensionless parameters defined

$$
\begin{aligned}
& \tilde{g}_{0}=\pi g_{0} \Lambda^{2}, \\
& \tilde{g}_{\rho}=\pi g_{\rho} \Lambda^{-2 \rho+2} .
\end{aligned}
$$

The $\beta$ functions that we have derived, Eqs. (27) and (??), describe how the two couplings - $g_{0}$ and $g_{\rho}$ - behave, if we rescale all the lengths and consider the elastic medium at coarser scales. If, for example, a small $g_{0}$ diverges under the RG rescaling, its implication is that a small $g_{0}$ at small length scales behaves as very strong disorder at much larger scales. Therefore, under such
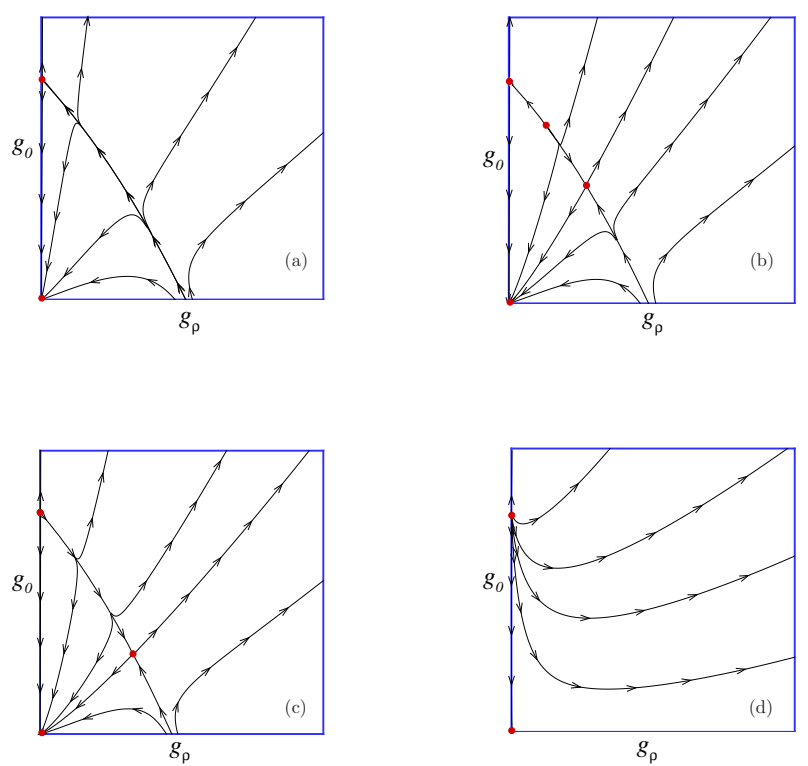

FIG. 4: Renoemalization group flows for (a) $\rho<0.14$; (b) $0.14<\rho<0.18$; (c) $0.18<\rho<1$, and (d) $\rho>1$.

condition, every wave amplitude will be localized. If, on the other hand, for some $g_{0}<g_{c}$ (where $g_{c}$ is a critical value of $\left.g_{0}\right) g_{0}$ vanishes under the RG rescaling, it implies that, in this regime, $g_{0}$ does not contribute much to the behavior of the propagating waves at large length scales. Therefore, one way of defining a localized state may be as follows: The waves are localized if, under the $\mathrm{RG}$ rescaling, at least either $g_{0}$ or $g_{\rho}$ diverges.

We must also point out that, one may begin the RG rescaling and analysis with the assumption that the couplings $g_{0}$ and $g_{\rho}$ are small. If, under the RG rescaling, we find stable fixed points (FPs), it would imply that the assumption of the couplings being small about such FPs is still valid. However, around an unstable FP the couplings can grow and, hence, the perturbation expansion that we have developed would fail. For our main purpose, however, namely, determining the localized/extended regimes and the transition between them, the most important goal is to determine the condition(s) under which the FPs are unstable, around which the couplings can diverge.

The FPs of the model are the roots of $\beta$ functions. The RG equations, together with the parameter $\rho$, have a complex phase space. Depending on $\rho$, there are four regimes:

(i) For $\rho<(-17557527+$ $128 \sqrt{19977620601}) / 3888601 \simeq 0.14$ there are two FPs: The trivial Gaussian FP, $\left\{\tilde{g}_{0}^{*}=\tilde{g}_{\rho}^{*}=0\right\}$, which is stable, and a nontrivial FP, $\left\{\tilde{g}_{0}^{*}=36 / 1585 \simeq 0.022, \tilde{g}_{\rho}^{*}=0\right\}$ which has one positive eigenvalue (along the eigendirection of which is unstable) and one negative one (along the eigendirection direction of which is stable); see Fig. 4a. Physically, this implies that the diagram is divided into two parts. In one part the Gaussian FP is relevant 
and the disorder does not have any effect, so that all the states are delocalized. In the second part, the values of couplings increase under rescaling, so that the disorder (both random and correlated) is relevant and, therefore, the elastic waves are localized. Thus, the line (more precisely, the curve) that separates the two parts is where the localization-delocalization transition takes place.

(ii) Four FPs exist if $0.14<\rho<289 / 1585 \simeq 0.18$. The Gaussian FP is stable. The other FPs are unstable in one eigendirection but stable in the other eigendirection, except, $\left\{\tilde{g}_{0}^{*}=0.022, \tilde{g}_{\rho}^{*}=0\right\}$, which has positive eigenvalues and, hence, is unstable in all directions. This is shown in Fig. 4b.

(iii) There are three FPs for $0.18<\rho<1$. The Gaussian FP is again stable. The FP, $\left\{g_{0}^{*}=0.022, g_{\rho}^{*}=0\right\}$ is unstable in all directions The third FP is unstable in one eigendirection but unstable in the second eigendirection. Figure 4c presents this part of the RG flow diagram.

In both (ii) and (iii), as $\rho$ increases, the system tries to move away from case (i) (the delocalized-localized transition) to a purely localized state (see also below). Moreover, in (i) - (iii) there is a point on the $g_{\rho}$ axis which obviously is not a FP, but the RG flows change their direction on the $g_{\rho}$ axis at that point. This means that one of the $\beta$ functions is zero on this axis, while the other one is not.

(iv) For $\rho>1$ there are two FPs. As Fig. 4d indicates, the Gaussian FP is stable on the $\tilde{g}_{0}$ axis but unstable on the $\tilde{g}_{\rho}$ axis, and the nontrivial FP, $\left\{\tilde{g}_{0}^{*}=0.022, \tilde{g}_{\rho}^{*}=\right.$ $0\}$, is unstable in all directions. The implication is that, while the power-law correlated disorder is relevant, no new FP exists to one-loop order and, therefore, the longwavelength behavior of the system is determined by the long-range component of the disorder. This means that for $\rho>1$ the elastic waves are localized in 2D.

Let us note that the extension of the present RG analysis to 3D systems is difficult, but doable. The reason for the difficulty is twofold. (i) It is difficult to determine the transformation matrix $\mathbf{U}$ [see Eq. (15)] for a 3D system, as its forms becomes very complex in 3D. Knowledge of $\mathbf{U}$ is necessary for diagonalizing the relevant matrices. (iii) As the Appendix indicates, the number of contribution Feynman diagrams is large is in 2D. The number of such diagrams much larger in 3D.

\section{COMPARISON WITH ACOUSTIC WAVE PROPAGATION}

Since scalar equations have often been invoked for describing propagation of elastic waves, it is of interest to compare the above results with those that we derived previously $^{25,26}$ for the scalar model of (acoustic) wave propagation in heterogeneous media with precisely the same type of disorder as what we consider in the present paper. The governing equation for such waves is given by

$$
m \frac{\partial^{2} u}{\partial t^{2}}=\boldsymbol{\nabla} \cdot[\lambda(\mathbf{x}) \nabla u(\mathbf{x})] .
$$

The analysis was carried out ${ }^{25,26}$ for a $d$-dimensional system, but we summarize its results for $2 \mathrm{D}$ media. The RG analysis indicated that, depending on $\rho$, there can be two distinct regimes (unlike the four regimes described above):

(i) For $0<\rho<1$ there are three sets of FPs. One set represents the Gaussian FP, $\left\{g_{0}^{*}=g_{\rho}^{*}=0\right\}$, which is stable. The other two are $\left\{g_{0}^{*}=1 / 4, g_{\rho}^{*}=0\right\}$, and [31]

$$
\begin{aligned}
& g_{0}^{*}=-\left[\frac{4}{11} d+\frac{5}{44}(2 \rho-d)\right]+\sqrt{\left[\frac{4}{11} d+\frac{5}{44}(2 \rho-d)\right]^{2}+\frac{5}{167}(2 \rho-2)^{2}}, \\
& g_{\rho}^{*}=\frac{3}{4} g_{0}^{*}+\frac{1}{16}(d-2 \rho),
\end{aligned}
$$

which, for $d=2$, reduces to

$$
\begin{aligned}
& g_{0}^{*}=-\frac{1}{22}(5 \rho+11)+\sqrt{\left[\frac{8}{11}+\frac{5}{11}(\rho-1)^{2}\right]^{2}+\frac{5}{167}(2 \rho-2)^{2}}, \\
& g_{\rho}^{*}=\frac{3}{4} g_{0}^{*}+\frac{1}{8}(1-\rho)
\end{aligned}
$$

which is stable in one eigendirection but unstable in the

other eigendirection. Therefore, for $0<\rho<1$ the one- 
loop RG analysis indicated that a medium with uncorrelated disorder is unstable against long-range correlated disorder towards a new FP in the space of the coupling constants. Hence, there is a phase transition from delocalized to localized acoustic waves with increasing the disorder intensity.

Thus, the physical implication of the $R G$ results for acoustic wave propagation described by Eq. (31) is as follows. In the interval, $0<\rho<1$, there is a region in the space of the coupling constants $\left\{g_{0}, g_{\rho}\right\}$ in which the RG flows take any initial point to the Gaussian FP. This implies that, for $0<\rho<1$, a disordered medium of the type considered in this paper and our previous work ${ }^{25,26}$ looks like a pure (ordered or homogeneous) medium at large length scales, implying that acoustic waves are $e x$ tended or delocalized.

However, when $g_{0}$ or $g_{\rho}$ are large enough that the initial point is out of the basin of attraction of the Gaussian FP, the RG flows move such points toward large values, hence implying that, under the RG rescaling, the probability density function of the disorder becomes broader and broader at increasingly larger length scales. Therefore, in this case, a propagating acoustic wave samples a medium with very large spatial fluctuations in the elastic stiffness or moduli. We also found that ${ }^{25,26}$ even if one starts in a disordered medium with purely long-range correlations (i.e., one with $g_{0}=0$ ), the RG equations indicate that the growth of $g_{\rho}$ will lead to increasing, i.e., nonzero, $g_{0}$, hence implying that uncorrelated disorder will be produced by the rescaling. Since the local fluctuations in the bulk moduli play the role of scattering points, the implication for acoustic waves is that the multiple scattering of a propagating wave from the uncorrelated disorder will destroy the wave's coherence, leading eventually to the localization of acoustic waves.

(ii) For $\rho>1$ there are two FPs: the Gaussian FP which is stable on the $g_{0}$ axis but not on the $g_{\rho}$ axis, and a second FP, $\left\{g_{0}^{*}=1 / 4, g_{\rho}^{*}=0\right\}$, which is unstable in all directions. The implication for acoustic waves is that, although power-low correlated disorder is relevant, no new FP exists to one-loop order and, therefore, the system's long-wavelength behavior is determined by the long-range component of the disorder. This implies that for $\rho>1$ the acoustic waves are localized (in fact, in this case they are localized for any $d$ ), which is similar to the elastic waves studied in the present paper. In addition, in both cases the system undergoes a disorder-induced transition when only the uncorrelated disorder is present.

Let us note that we argued in our previous papers ${ }^{25,26}$ that, in the case of acoustic waves, although, similar to the elastic waves considered in the present paper, the RG calculations were carried out to one-loop order, the analysis should still be valid for higher orders of the perturbation as well. The argument was based on the fact that the signs of the higher-order terms are all positive. That this is so is due to the following. We must keep in mind that the contraction coefficients for auxiliary fields are always greater than those of auxiliary and Grassma- nian fields that supply the negative terms. Moreover, the numbers of diagrams of, e.g., a real auxiliary field and an imaginary auxiliary field are equal to number of diagrams of an auxiliary and Grassmanian field. This implies immediately that the signs of higher-order terms should also be positive. We, therefore, concluded that ${ }^{25,26}$ the oneloop results for the acoustic waves should be valid to all orders. However, we now believe that this is only a necessary but not sufficient conditions. In the case of elastic waves, though, we cannot even determine a priori the signs of the higher-order terms.

Thus, comparison of propagation of elastic and acoustic waves in the type of heterogeneous media that we consider in this paper indicates that, while the RG flow diagrams for the elastic waves is more complex than those of the acoustic waves, the region of the coupling constants space in which they are delocalized is narrower than that of the acoustic waves.

\section{SUMMARY}

We developed a field-theoretic description of propagation and localization of elastic waves in 2D heterogeneous solids using a RG approach. Two types of heterogeneities, random disorder and one with long-range correlations with a power-law correlation function, were considered. We found that in presence of power-law correlated disorder with the exponent $\rho>1$ (non-decaying correlations) the RG flows are toward the strong coupling regime, and the waves are localized. For $\rho<1$, and depending on its value, there are other fixed points. One, which is stable, is the Gaussian FP with a small domain of attraction. In this domain, long-range correlated disorder, as well as the random disorder, are irrelevant and, therefore, the waves are delocalized. In this regard, the delocalized states in the Gaussian domain are unlike electrons in 2D systems, which remain localized for any disorder.

Whether the delocalized states predicted for the Gaussian domain persist, if we analyze the RG flows beyond the one-loop approximation, remains to be seen. It may be that the domain of attraction of the Gaussian FP shrinks (and might disappear completely), if we consider the contributions of the higher order loops. However, analytical determination of the contribution of even the second-order loops for this problem is very difficult.

As we mentioned in the Introduction, a challenging feature of the localization problem is obtaining an analytical estimate of the localization length exponent. In this regard, the previous analytical approaches are in contradiction with the numerical results. We hope that the method developed in this paper can provide a precise way of describing the critical properties of the localizationdelocalization transition and its critical exponents in higher dimensions.

We are currently carrying out extensive numerical simulations in order to further check the accuracy of the predictions of the dynamical RG method developed in this paper. The results will be reported in the near future. 


\section{ACKNOWLEDGMENTS}

The work of R.S. was supported by the NIOC. We thank Mehdi Vaez Allaei for his valuable help in the preparation of the manuscript.

\section{Appendix: Integrals for the Feynman Diagrams}

In this Appendix we list all the expressions for the Feynman diagrams shown in Fig. 3.

$$
\begin{aligned}
& a_{1}=\int \frac{d^{2} q}{\left(q^{2}-\frac{\omega^{2}}{\lambda_{0}}\right)^{2}} \times \frac{1}{4}\left[A\left(p_{1}, p_{2}\right) A(-q, q)+A\left(q, p_{2}\right) A\left(p_{1},-q\right)\right] \\
& \times\left[A\left(p_{3}, p_{4}\right) A(q,-q)+A\left(q, p_{4}\right) A\left(p_{3},-q\right)\right] . \\
& =\frac{1}{4} p_{1} p_{2} p_{3} p_{4} \int q d q \int d \theta\left[9+\left(3 \cos ^{2} \theta+\sin ^{2} \theta\right)^{2}\right]^{2}=\frac{1523 \pi}{16} p_{1} p_{2} p_{3} p_{4} \int q d q \text {. } \\
& a_{2}=\int \frac{d^{2} q}{\left(q^{2}-\frac{\omega^{2}}{\lambda_{0}}\right)^{2}} \times 4\left[A\left(p_{2}, p_{1}\right) A(q,-q)\right]\left[A\left(p_{4}, p_{3}\right) A(q,-q)\right] . \\
& =324 p_{1} p_{2} p_{3} p_{4} \int q d q \int d \theta=648 \pi p_{1} p_{2} p_{3} p_{4} \int q d q \text {. } \\
& a_{3}=-\int \frac{d^{2} q}{\left(3 q^{2}-\frac{\omega^{2}}{\lambda_{0}}\right)\left(q^{2}-\frac{\omega^{2}}{\lambda_{0}}\right)} \times \frac{1}{2}\left[A\left(-q, p_{1}\right) C\left(p_{2}, q\right)\right]\left[A\left(p_{4}, q\right) C\left(-q, p_{3}\right)\right] \text {. } \\
& =\frac{1}{3} p_{1} p_{2} p_{3} p_{4} \int q d q \int d \theta\left(3 \cos ^{2} \theta+\sin ^{2} \theta\right)^{2} \sin ^{2}(2 \theta)=\frac{17 \pi}{12} p_{1} p_{2} p_{3} p_{4} \int q d q \text {. } \\
& a_{4}=\int \frac{d^{2} q}{\left(3 q^{2}-\frac{\omega^{2}}{\lambda_{0}}\right)^{2}} \times 4\left[A\left(p_{2}, p_{1}\right) B(-q, q)-C\left(p_{2}, q\right) C\left(-q, p_{1}\right)\right] \\
& \times\left[A\left(p_{4}, p_{3}\right) B(-q, q)+C\left(p_{4}, q\right) C\left(-q, p_{3}\right)\right] . \\
& =\frac{4}{9} p_{1} p_{2} p_{3} p_{4} \int q d q \int d \theta\left[3+\sin ^{2}(2 \theta)\right]^{2}=11 \pi p_{1} p_{2} p_{3} p_{4} \int q d q \text {. } \\
& a_{5}=\int \frac{d^{2} q}{\left(3 q^{2}-\frac{\omega^{2}}{\lambda_{0}}\right)^{2}} \times 4\left[A\left(p_{2}, p_{1}\right) B(-q, q)\right]\left[A\left(p_{4}, p_{3}\right) B(q,-q)\right] . \\
& =4 p_{1} p_{2} p_{3} p_{4} \int q d q \int d \theta=8 \pi p_{1} p_{2} p_{3} p_{4} \int q d q \text {. } \\
& a_{6}=\int \frac{d^{2} q}{\left(q^{2}-\frac{\omega^{2}}{\lambda_{0}}\right)^{2}} \times \frac{1}{4}\left[A\left(q, p_{1}\right) A\left(-q, p_{3}\right)+A\left(-q, p_{1}\right) A\left(q, p_{3}\right)\right] \\
& \times\left[A\left(p_{2},-q\right) A\left(p_{4}, q\right)+A\left(p_{4},-q\right) A\left(p_{2}, q\right)\right] . \\
& =p_{1} p_{2} p_{3} p_{4} \int q d q \int d \theta\left(3 \cos ^{2} \theta+\sin ^{2} \theta\right)^{2}=9 \pi p_{1} p_{2} p_{3} p_{4} \int q d q \text {. } \\
& a_{7}=-\int \frac{d^{2} q}{\left(3 q^{2}-\frac{\omega^{2}}{\lambda_{0}}\right)\left(q^{2}-\frac{\omega^{2}}{\lambda_{0}}\right)} \times 4\left[A\left(q, p_{1}\right) C\left(-q, p_{3}\right)\right]\left[A\left(p_{2},-q\right) C\left(p_{4}, q\right)\right] \text {. } \\
& =\frac{4}{3} p_{1} p_{2} p_{3} p_{4} \int q d q \int d \theta=\frac{8 \pi}{3} p_{1} p_{2} p_{3} p_{4} \int q d q \text {. } \\
& a_{8}=\int \frac{d^{2} q}{3\left(q^{2}-\frac{\omega^{2}}{\lambda_{0}}\right)\left(3 q^{2}-\frac{\omega^{2}}{\lambda_{0}}\right)} \times \frac{1}{4}\left[C\left(q, p_{1}\right) C\left(-q, p_{3}\right)+C\left(-q, p_{1}\right) C\left(q, p_{3}\right)\right] \\
& \times\left[C\left(p_{2},-q\right) C\left(p_{4}, q\right)+C\left(p_{4},-q\right) C\left(p_{2}, q\right)\right] . \\
& =\frac{1}{9} p_{1} p_{2} p_{3} p_{4} \int q d q \int d \theta \sin ^{4}(2 \theta)=\frac{\pi}{12} p_{1} p_{2} p_{3} p_{4} \int q d q \text {. }
\end{aligned}
$$


11

$$
\begin{aligned}
& a_{9}=a_{10}=-\int \frac{d^{2} q}{\left(q^{2}-\frac{\omega^{2}}{\lambda_{0}}\right)^{2}} \times 4\left[A\left(p_{2}, p_{1}\right) A(q,-q)\right]\left[A\left(p_{4}, p_{3}\right) A(q,-q)\right] . \\
& =-324 p_{1} p_{2} p_{3} p_{4} \int q d q \int d \theta=-648 \pi p_{1} p_{2} p_{3} p_{4} \int q d q \text {. } \\
& a_{11}=a_{12}=-\int \frac{d^{2} q}{\left(3 q^{2}-\frac{\omega^{2}}{\lambda_{0}}\right)^{2}} \times 4\left[A\left(p_{2}, p_{1}\right) B(q,-q)\right]\left[A\left(p_{4}, p_{3}\right) B(q,-q)\right] . \\
& =-4 p_{1} p_{2} p_{3} p_{4} \int q d q \int d \theta=-8 \pi p_{1} p_{2} p_{3} p_{4} \int q d q . \\
& b_{1}=\int \frac{d^{2} q}{\left(q^{2}-\frac{\omega^{2}}{\lambda_{0}}\right)^{2}} \times \frac{1}{2}\left[A\left(p_{2},-q\right) A\left(p_{1}, q\right)\right]\left[A\left(p_{4}, p_{3}\right) A(q,-q)+A\left(q, p_{3}\right) A\left(p_{4},-q\right)\right] . \\
& =\frac{1}{2} p_{1} p_{2} p_{3} p_{4} \int q^{-2 \rho+1} d q \int d \theta\left[9+\left(3 \cos ^{2} \theta+\sin ^{2} \theta\right)^{2}\right]\left(3 \cos ^{2} \theta+\sin ^{2} \theta\right)^{2} \\
& =\frac{551 \pi}{8} p_{1} p_{2} p_{3} p_{4} \int q^{-2 \rho+1} d q \text {. } \\
& b_{2}=b_{3}=\int \frac{d^{2} q}{\left(q^{2}-\frac{\omega^{2}}{\lambda_{0}}\right)^{2}} \times \frac{1}{4}\left[A\left(p_{2}, q\right) A\left(p_{4},-q\right)+A\left(p_{4}, q\right) A\left(p_{2},-q\right)\right] \\
& \times\left[A\left(p_{1}, q\right) A\left(p_{3},-q\right)+A\left(p_{1},-q\right) A\left(p_{3}, q\right)\right] . \\
& =p_{1} p_{2} p_{3} p_{4} \int q^{-2 \rho+1} d q \int d \theta\left(3 \cos ^{2} \theta+\sin ^{2} \theta\right)^{2}=9 \pi p_{1} p_{2} p_{3} p_{4} \int q^{-2 \rho+1} d q \text {. } \\
& b_{4}=\int \frac{d^{2} q}{3\left(q^{2}-\frac{\omega^{2}}{\lambda_{0}}\right)\left(3 q^{2}-\frac{\omega^{2}}{\lambda_{0}}\right)} \times \frac{1}{4}\left[C\left(q, p_{1}\right) C\left(-q, p_{3}\right)+C\left(-q, p_{1}\right) C\left(q, p_{3}\right)\right] \\
& \times\left[C\left(p_{2},-q\right) C\left(p_{4}, q\right)+C\left(p_{4},-q\right) C\left(p_{2}, q\right)\right] . \\
& =\frac{1}{9} p_{1} p_{2} p_{3} p_{4} \int q^{-2 \rho+1} d q \int d \theta \sin ^{4}(2 \theta)=\frac{\pi}{12} p_{1} p_{2} p_{3} p_{4} \int q^{-2 \rho+1} d q . \\
& b_{5}=-\int \frac{d^{2} q}{3\left(q^{2}-\frac{\omega^{2}}{\lambda_{0}}\right)\left(q^{2}-\frac{\omega^{2}}{\lambda_{0}}\right)} \times\left[A\left(p_{4},-q\right) C\left(q, p_{3}\right)\right]\left[A\left(q, p_{1}\right) C\left(p_{2},-q\right)\right] . \\
& =\frac{2}{3} p_{1} p_{2} p_{3} p_{4} \int q^{-2 \rho+1} d q \int d \theta \sin ^{2}(2 \theta)\left(3 \cos ^{2} \theta+\sin ^{2} \theta\right)^{2}=\frac{17 \pi}{6} p_{1} p_{2} p_{3} p_{4} \int q^{-2 \rho+1} d q . \\
& b_{6}=-\int \frac{d^{2} q}{\left(3 q^{2}-\frac{\omega^{2}}{\lambda_{0}}\right)\left(q^{2}-\frac{\omega^{2}}{\lambda_{0}}\right)} \times 2\left[A\left(q, p_{3}\right) C\left(p_{4},-q\right)\right]\left[A\left(p_{2},-q\right) C\left(q, p_{1}\right)\right] . \\
& =\frac{2}{3} p_{1} p_{2} p_{3} p_{4} \int q^{-2 \rho+1} d q \int d \theta \sin ^{2}(2 \theta)\left(3 \cos ^{2} \theta+\sin ^{2} \theta\right)^{2}=\frac{17 \pi}{6} p_{1} p_{2} p_{3} p_{4} \int q^{-2 \rho+1} d q \text {. } \\
& b_{7}=-\int \frac{d^{2} q}{\left(3 q^{2}-\frac{\omega^{2}}{\lambda_{0}}\right)\left(q^{2}-\frac{\omega^{2}}{\lambda_{0}}\right)} \times 4\left[A\left(q, p_{1}\right) C\left(-q, p_{3}\right)\right]\left[A\left(p_{2},-q\right) C\left(p_{4}, q\right)\right] . \\
& =\frac{4}{3} p_{1} p_{2} p_{3} p_{4} \int q^{-2 \rho+1} d q \int d \theta \sin ^{2}(2 \theta)\left(3 \cos ^{2} \theta+\sin ^{2} \theta\right)^{2}=\frac{17 \pi}{3} p_{1} p_{2} p_{3} p_{4} \int q^{-2 \rho+1} d q . \\
& b_{8}=\int \frac{d^{2} q}{\left(3 q^{2}-\frac{\omega^{2}}{\lambda_{0}}\right)\left(3 q^{2}-\frac{\omega^{2}}{\lambda_{0}}\right)} \times 4\left[A\left(p_{4}, p_{3}\right) B(q,-q)-C\left(q, p_{3}\right) C(p 4,-q)\right]\left[C\left(p_{2},-q\right) C\left(q, p_{1}\right)\right] . \\
& =\frac{4}{9} p_{1} p_{2} p_{3} p_{4} \int q^{-2 \rho+1} d q \int d \theta\left[3+\sin ^{2}(2 \theta)\right] \sin ^{2}(2 \theta)=\frac{15 \pi}{3} p_{1} p_{2} p_{3} p_{4} \int q^{-2 \rho+1} d q . \\
& c_{1}=\int \frac{d^{2} q}{\left(q^{2}-\frac{\omega^{2}}{\lambda_{0}}\right)^{2}} \times\left[A\left(q, p_{1}\right) A\left(p_{2},-q\right)\right]\left[A\left(p_{3},-q\right) A\left(q, p_{4}\right)\right] . \\
& =p_{1} p_{2} p_{3} p_{4} \int q^{-4 \rho+1} d q \int d \theta\left(3 \cos ^{2} \theta+\sin ^{2} \theta\right)^{4}=\frac{227 \pi}{4} p_{1} p_{2} p_{3} p_{4} \int q^{-4 \rho+1} d q . \\
& c_{2}=\int \frac{d^{2} q}{\left(q^{2}-\frac{\omega^{2}}{\lambda_{0}}\right)^{2}} \times\left[A\left(q, p_{1}\right) A\left(-q, p_{3}\right)\right]\left[A\left(p_{2},-q\right) A\left(p_{4}, q\right)\right] . \\
& =p_{1} p_{2} p_{3} p_{4} \int q^{-4 \rho+1} d q \int d \theta\left(3 \cos ^{2} \theta+\sin ^{2} \theta\right)^{4}=\frac{227 \pi}{4} p_{1} p_{2} p_{3} p_{4} \int q^{-4 \rho+1} d q . \\
& c_{3}=\int \frac{d^{2} q}{\left(3 q^{2}-\frac{\omega^{2}}{\lambda_{0}}\right)^{2}} \times 4\left[C\left(q, p_{1}\right) C\left(p_{2},-q\right)\right]\left[C\left(p_{4},-q\right) C\left(q, p_{3}\right)\right] . \\
& =\frac{4}{9} p_{1} p_{2} p_{3} p_{4} \int q^{-4 \rho+1} d q \int d \theta \sin ^{4}(2 \theta)=\frac{\pi}{3} p_{1} p_{2} p_{3} p_{4} \int q^{-4 \rho+1} d q .
\end{aligned}
$$




$$
\begin{aligned}
& c_{4}=\int \frac{d^{2} q}{\left(3 q^{2}-\frac{\omega^{2}}{\lambda_{0}}\right)\left(q^{2}-\frac{\omega^{2}}{\lambda 0}\right)} \times(-4)\left[A\left(q, p_{1}\right) C\left(p_{2},-q\right)\right]\left[A\left(p_{4},-q\right) C\left(q, p_{3}\right)\right] . \\
& =p_{1} p_{2} p_{3} p_{4} \times \frac{4}{3} \int q^{-4 \rho+1} d q \int d \theta\left(3 \cos ^{2} \theta+\sin ^{2} \theta\right)^{2} \sin ^{2}(2 \theta)=\frac{17 \pi}{3} p_{1} p_{2} p_{3} p_{4} \int q^{-4 \rho+1} d q \text {. } \\
& c_{5}=\int \frac{d^{2} q}{\left(3 q^{2}-\frac{\omega^{2}}{\lambda_{0}}\right)\left(q^{2}-\frac{\omega^{2}}{\lambda_{0}}\right)} \times(-4)\left[A\left(q, p_{1}\right) C\left(-q, p_{3}\right)\right]\left[A\left(p_{2},-q\right) C\left(p_{4}, q\right)\right] . \\
& =\frac{4}{3} p_{1} p_{2} p_{3} p_{4} \int q^{-4 \rho+1} d q \int d \theta\left(3 \cos ^{2} \theta+\sin ^{2} \theta\right)^{2} \sin ^{2}(2 \theta)=\frac{17 \pi}{3} p_{1} p_{2} p_{3} p_{4} \int q^{-4 \rho+1} d q \text {. } \\
& c_{6}=\int \frac{d^{2} q}{\left(3 q^{2}-\frac{\omega^{2}}{\lambda_{0}}\right)\left(3 q^{2}-\frac{\omega^{2}}{\lambda_{0}}\right)} \times\left[C\left(q, p_{1}\right) C\left(-q, p_{3}\right)\right]\left[C\left(p_{2},-q\right) C\left(p_{4}, q\right)\right] . \\
& =\frac{1}{9} p_{1} p_{2} p_{3} p_{4} \int q^{-4 \rho+1} d q \int d \theta \sin ^{4}(2 \theta)=\frac{\pi}{12} p_{1} p_{2} p_{3} p_{4} \int q^{-4 \rho+1} d q \text {. } \\
& d_{1}=\int \frac{d^{2} q}{\left(q^{2}-\frac{\omega^{2}}{\lambda_{0}}\right)^{2}} \times \frac{1}{2}\left[A\left(p_{2}, p_{1}\right) A(-q, q)+A\left(-q, p_{1}\right) A\left(p_{2}, q\right)\right]\left[A\left(p_{4}, p_{3}\right) A(q,-q)\right] \text {. } \\
& =\frac{9}{2} p_{1} p_{2} p_{3} p_{4} \int q d q \int d \theta\left[9+\left(3 \cos ^{2} \theta+\sin ^{2} \theta\right)^{2}\right]=\frac{243 \pi}{2} p_{1} p_{2} p_{3} p_{4} \int q d q \text {. } \\
& d_{2}=\int \frac{d^{2} q}{\left(q^{2}-\frac{\omega^{2}}{\lambda_{0}}\right)^{2}} \times 4\left[A\left(p_{2}, p_{1}\right) A(-q, q)\right]\left[A\left(p_{4}, p_{3}\right) A(q,-q)\right] . \\
& =324 p_{1} p_{2} p_{3} p_{4} \int q d q \int d \theta=324 \pi p_{1} p_{2} p_{3} p_{4} \int q d q . \\
& d_{3}=d_{4}=0 \text {. } \\
& d_{5}=d_{6}=\int \frac{d^{2} q}{\left(3 q^{2}-\frac{\omega^{2}}{\lambda_{0}}\right)^{2}} \times 4\left[A\left(p_{2}, p_{1}\right) B(q,-q)\right]\left[A\left(p_{3}, p_{4}\right) A(q,-q)\right] . \\
& =4 p_{1} p_{2} p_{3} p_{4} \int q d q \int d \theta=8 \pi p_{1} p_{2} p_{3} p_{4} \int q d q \text {. } \\
& d_{7}=d_{8}=-\int \frac{d^{2} q}{\left(3 q^{2}-\frac{\omega^{2}}{\lambda_{0}}\right)^{2}} \times 4\left[A\left(p_{2}, p_{1}\right) B(q,-q)\right]\left[A\left(p_{4}, p_{3}\right) A(-q, q)\right] \text {. } \\
& =-324 p_{1} p_{2} p_{3} p_{4} \int q d q \int d \theta=-648 \pi p_{1} p_{2} p_{3} p_{4} \int q d q \text {. } \\
& d_{9}=d_{10}=0 \text {. } \\
& e_{1}=\int \frac{d^{2} q}{\left(q^{2}-\frac{\omega^{2}}{\lambda_{0}}\right)^{2}} \times\left[A\left(q, p_{1}\right) A\left(p_{2},-q\right)\right]\left[A\left(p_{4}, p_{3}\right) A(q,-q)\right] \text {. } \\
& =9 p_{1} p_{2} p_{3} p_{4} \int q^{-2 \rho+1} d q \int d \theta\left(3 \cos ^{2} \theta+\sin ^{2} \theta\right)^{2}=81 \pi p_{1} p_{2} p_{3} p_{4} \int q^{-2 \rho+1} d q \text {. } \\
& e_{2}=e_{3}=0 \text {. } \\
& e_{4}=\int \frac{d^{2} q}{\left(3 q^{2}-\frac{\omega^{2}}{\lambda_{0}}\right)^{2}} \times(-4)\left[C\left(q, p_{1}\right) C\left(p_{2},-q\right)\right]\left[A\left(p_{4}, p_{3}\right) B(q,-q)\right] \text {. } \\
& =\frac{4}{3} p_{1} p_{2} p_{3} p_{4} \int q^{-2 \rho+1} d q \int d \theta \sin ^{2}(2 \theta)=\frac{4 \pi}{3} p_{1} p_{2} p_{3} p_{4} \int q^{-2 \rho+1} d q \text {. }
\end{aligned}
$$

*moe@iran.usc.edu

${ }^{1}$ P. W. Anderson, Phys. Rev. 109, 1492 (1958); N. F. Mott and W. D. Twose, Adv. Phys. 10, 107 (1961).

${ }^{2}$ S. He and J. D. Maynard, Phys. Rev. Lett. 57, 3171
(1986); J. D. Maynard, Rev. Mod. Phys. 73, 401 (2001).

${ }^{3}$ D. S. Wiersma, P. Bartolini, A. Lagendijk, and R. Righini, Nature (London) 390, 671 (1997).

${ }^{4}$ E. Abrahams, P. W. Anderson, D. C. Licciardello, and T. V. Ramakrishnan, Phys. Rev. Lett. 42, 673 
(1979); P. W. Anderson, E. Abrahams, and T. V. Ramakrishnan, ibid. 43, 718 (1979).

${ }^{5}$ D. Vollhardt and P. Wölfle, Phys. Rev. B 22, 4666 (1980).

${ }^{6}$ T. R. Kirkpatrick, Phys. Rev. B 31, 5746 (1985); C. A. Condat and T. R. Kirkpatrick, ibid. 33, 3102 (1986); ibid. 36, 6782 (1987).

${ }^{7}$ F. J. Wegner, Z. Phys. B 25, 327 (1976); 35, 207 (1979); 36, 209 (1980); Nucl. Phys. B 180[FS2], 77 (1981).

${ }^{8}$ K. B. Efetov, A. I. Larkin, and D. E. Khmelnitskii, Sov. Phys. JETP 52, 568 (1980).

${ }^{9}$ S. Hikami, Phys. Rev. B 24, 2671 (1981).

${ }^{10}$ A. MacKinnon and B. Kramer, Z. Phys. B 53, 1 (1983); B. Kramer and A. MacKinnon, Rep. Prog. Phys. 56, 1469 (1993).

${ }^{11}$ P. C. Martin, E. D. Siggia, and H. A. Rose, Phys. Rev. A 8, 423 (1973).

${ }^{12}$ N. Bleistein, J. K. Cohen, and J. W. Stockwell, Jr., Mathematics of Multidimensional Seismic Imaging, Migration, and Inversion (Springer, New York, 2001); A. Ishimaru, Wave Propagation and Scattering in Random Media (Oxford University Press, Oxford, 1997).

${ }^{13}$ For recent experimental observation of weak localization of seismic waves see, for example, E. Larose, L. Margerin, B. A. van Tiggelen, and M. Campillo, Phys. Rev. Lett. 93, 048501 (2004).

${ }^{14}$ S. John, H. Sompolinsky, and M. J. Stephen, Phys. Rev. B 27, 5592 (1983).

${ }^{15}$ M. Sahimi, Heterogeneous Materials I (Springer, New York, 2003), chapters 6 and 9; P. Sheng, Introduction to Wave Scattering, Localization, and Mesoscopic Phenomena (Academic, San Diego, 1995).

${ }^{16}$ For reviews see, for example, Refs. [15] and, T. Nakayama, K. Yakubo, and R. Orbach, Rev. Mod. Phys. 66, 381 (1994).

${ }^{17}$ M. Belhadi, O. Rafil, R. Tigrine, A. Khater, A. Virlouvet, and K. Maschke, Eur. Phys. J. B 15, 435 (2004); J. J. Ludlam, S. N. Taraskin, and S. R. Elliot, Phys. Rev. B 67, 132203 (2003); J. J. Ludlam, T. O. Stadelmann, S. N. Taraskin, and S. R. Elliot, J. Non-Cryst. Solids 293-295, 676 (2001); D. García-Pablos, M. Sigalas, F. R. Montero de Espinosa, M. Torres, M. Kafesaki, and N. Garcia, Phys. Rev. Lett. 84, 4349 (2000).
${ }^{18}$ B. Garber, M. Cahay, and G. E. W. Bauer, Phys. Rev. B 62, 12831 (2000).

${ }^{19}$ F. M. Li, Y. S. Wang, C. Hu, and W. H. Huang, Waves in Random Media 14, 217 (2004).

${ }^{20}$ B. A. van Tiggelen, L. Margerin, and M. Campillo, J. Acous. Soc. Am. 110, 1291 (2001).

${ }^{21}$ L. Margerin, M. Campillo, and B. van Tiggelen, J. Geophys. Res. 105, 7873 (2000).

${ }^{22}$ See, for example, M. Bouchon, Pure Appl. Geophys. 160, 445 (2004); W. Sun and H. Yang, Acta Mech. Solida Sinica 16, 283 (2004); T.-K. Hong and B. L. N. Kennet, Geophys. J. International 154, 483 (2003); ibid. 150, 610 (2002); T. Bohlen, Comput. Geosci. 28, 887 (2002); H. A. Friis, T. A. Johansen, M. Haveraaen, H. Muthe-Kaas, and A. Drottning, Appl. Num. Math. 39, 151 (2001).

${ }^{23}$ M. Sahimi and S. E. Tajer, Phys. Rev. E 71, 046301 (2005).

${ }^{24}$ M. R. Rahimi Tabar, M. Sahimi, F. Ghasemi, K. Kaviani, M. Allamehzadeh, J. Peinke, M. Mokhtari, M. Vesaghi, M. D. Niry, A. Bahraminasab, S. Tabatabai, S. Fayyazbakhsh, and M. Akbari, in Modelling Critical and Catastrophic Phenomena in Geoscience, edited by P. Bhattacharyya and B. K. Chakrabarti (Springer, Berlin, 2006).

${ }^{25}$ F. Shahbazi, A. Bahraminasab, S. M. Vaez Allaei, M. Sahimi, and M. R. Rahimi Tabar, Phys. Rev. Lett. 94, 165505 (2005).

${ }^{26}$ A. Bahraminasab, S. M. Vaez Allaei, F. Shahbazi, M. Sahimi, M. D. Niry, and M. R. Rahimi Tabar, Phys. Rev. B 75, 064301 (2007).

${ }^{27}$ C. De Dominicis and L. Peliti, Phys. Rev. Lett. 38, 505 (1977); Phys. Rev. B 18, 353 (1978).

${ }^{28}$ D. Hochberg, C. Molina, J. Perez, and M. Visser, Phys. Rev. E 60, 6343 (1999).

${ }^{29}$ J. Cardy, Scaling and Renormalization in Statistical Physics (Cambridge University Press, London, 1996).

${ }^{30}$ N. Goldenfeld, Lectures on Phase Transitions and the Renormalization Group

(Addison-Wesley, New York, 1992).

${ }^{31}$ The formula that was given by Bahraminasab et al. ${ }^{26}$ for $g_{0}^{*}$ contains some minor errors in the numerical coefficients. Equation (32) provides the correct formula. 